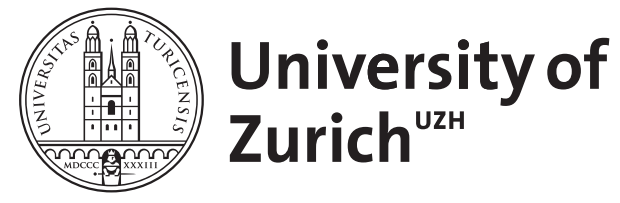

\title{
Zang-Hee Cho (Editor): 7.0 Tesla MRI Brain Atlas: In Vivo Atlas with Cryomacrotome Correlation
}

\author{
Kockro, Ralf A ; Amaxopoulou, C
}

DOI: https://doi.org/10.1007/s10143-010-0265-6

Posted at the Zurich Open Repository and Archive, University of Zurich ZORA URL: https://doi.org/10.5167/uzh-156691

Journal Article

Published Version

Originally published at:

Kockro, Ralf A; Amaxopoulou, C (2010). Zang-Hee Cho (Editor): 7.0 Tesla MRI Brain Atlas: In Vivo Atlas with Cryomacrotome Correlation. Neurosurgical Review, 33(3):383-384.

DOI: https://doi.org/10.1007/s10143-010-0265-6 


\title{
Zang-Hee Cho (Editor): 7.0 Tesla MRI Brain Atlas: In Vivo Atlas with Cryomacrotome Correlation
}

\author{
Springer 2010, 557 pages, 500 illustrations
}

\author{
Ralf A. Kockro • C. Amaxopoulou
}

Received: 3 May 2010/Revised: 3 May 2010/Accepted: 4 May 2010/Published online: 27 May 2010

(C) Springer-Verlag 2010

Advances in the area of ultra high field magnetic resonance imaging (MRI) have generated considerable attention in the field of neuroscience. This brain atlas, created by Zang-Hee Cho (Gachon University of Medicine and Science, Korea) and colleagues, presents a corresponding display of high field T2-weighted in vivo images and cryomacrotome images on an impressive $15.1 \times 12.1$ in. page format. The MRI images were acquired from a 27 -year-old male volunteer in 2D gradient echo T2 technique by a Siemens Magnetom 7 T MR scanner and reconstructed on a Siemens Avanto series console. The cryotome slices were obtained from a 67-year-old male in 2-mm slice thickness. Subdivided in three chapters, the images are presented in an axial, coronal, and sagittal orientation. Viewing the open atlas, each right side displays an MRI, and each left side, the corresponding photographic cadaver section. Since both series do not originate from the same person, the images are displayed in an approximately matched spatial orientation in order to illustrate similar anatomical structures. All images are labeled with lines pointing to specific structures and millimeter scales allow spatial measurements in a Cartesian coordinate system. Almost every third image displays a magnified view, depicting areas like the deep brain, midbrain, and brainstem.

At first glance, the atlas impresses with its format. The 1:1 scale display enables accurate examination of brain anatomy in authentic dimensions and down to the level of

\footnotetext{
R. A. Kockro $(\bowtie)$

Department of Neurosurgery, University Hospital,

Zürich, Switzerland

e-mail: ralfalfons.kockro@usz.ch

C. Amaxopoulou

Department of Neuroradiology, University Hospital,

Zürich, Switzerland
}

minute structures. The $7 \mathrm{~T}$ images especially impress with their axial and sagittal depiction of the deep brain, such as thalamic nuclei, basal ganglia, anterior and posterior commissure, fornix, subdivision of the internal capsule, optic radiation, substantia nigra, red nucleus, and transcortical and medullary vasculature. On the expanded view of the hippocampus, fine details such as the subiculum and the dentate gyrus are visible. Some artifacts are noticeable on the sagittal images; however, they hardly diminish the information displayed. All cranial nerves except for the accessory nerve are visible on the MRI; however, one would expect them to be displayed in even higher contrast level. Since the atlas does not display the skull base, the cranial nerves are truncated a few millimeters from their brain stem exit. This is a pity, since the combined display of cranial nerves and skull base, particularly along the cavernous sinus, would be interesting information for skull base surgery teaching and planning. For functional neurosurgery, the atlas presents a valuable tool since it pushes the boundaries of deep brain structural imaging by directly visualizing functional targets like the subthalamic or ventralis intermedius nucleus and by simultaneously illustrating the anatomical correlation on the cryotome images. Furthermore, the Cartesian scales on the sides of the pages enable precise understanding of spatial dimensions.

In the area of the brain stem, the atlas falls a little behind expectations since structures like the corticospinal tract, medial lemniscus, medial longitudinal fasciculus, or cranial nerve nuclei are often difficult to differentiate on the MRI planes, despite labeling lines pointing to them. Furthermore, the corresponding anatomical cross-sections do not match consistently. In this area, Duvernoy's Atlas of the Human Brain Stem and Cerebellum by Naidich T.P., Duvernoy H.M., Delman B.N., Sorensen A.G., Kollias S. S., and Haacke E.M., which is based on 9.4 T MRI and 
selected anatomical preparations, appears significantly more detailed and informative. From a neurosurgical perspective, high-resolution brain stem imaging, including clear depiction of white matter tracks and cranial nerve nuclei, especially with neighboring pathology is the essential gateway to demystify surgical procedures in this area.

The authors are suggesting to establish a data collection system for "clinicopathologic brain mapping" by using the atlas' anterior-posterior commissure-related image numbering and Cartesian coordinate system. The authors are prepared to receive MRI data accompanied by clinical information recorded on a specific from across the world in order to build a database correlating clinical symptoms and findings on MRI. This is a commendable effort since a detailed clinicopathological map would certainly be of significant value for both neuroscientists and clinicians.

This 7.0 Tesla MRI atlas is an innovative piece of work integrating the latest techniques of ultra high field MRI and detailed neuroanatomy preparations. Since the series originate from two different individuals, the problem of accurate matching could only be overcome by obtaining high field MRI data from a volunteer who would be willing to donate his body for postmortem cryo-sectioning. This would lead to perfectly matched data for a future atlas. For neurosurgeons, neuroradiologists, anatomists, and students, the atlas by ZangHee Cho and his colleagues provides comprehensive structural information and serves as a review for both basic and more detailed anatomy. It is certainly an enrichment for every neuroscience library. 